\title{
Defining complete and observable chaos
}

\author{
by Víctor Jiménez López (Murcia)
}

\begin{abstract}
For a continuous map $f$ from a real compact interval $I$ into itself, we consider the set $\mathrm{C}(f)$ of points $(x, y) \in I^{2}$ for which $\liminf _{n \rightarrow \infty}\left|f^{n}(x)-f^{n}(y)\right|=0$ and $\limsup _{n \rightarrow \infty}\left|f^{n}(x)-f^{n}(y)\right|>0$. We prove that if $\mathrm{C}(f)$ has full Lebesgue measure then it is residual, but the converse may not hold. Also, if $\lambda^{2}$ denotes the Lebesgue measure on the square and $\operatorname{Ch}(f)$ is the set of points $(x, y) \in \mathrm{C}(f)$ for which neither $x$ nor $y$ are asymptotically periodic, we show that $\lambda^{2}(\mathrm{C}(f))>0$ need not imply $\lambda^{2}(\mathrm{Ch}(f))>0$. We use these results to propose some plausible definitions of "complete" and "observable" chaos.
\end{abstract}

1. Introduction. In what follows, $I$ will be a compact real interval and $C(I)$ will denote the set of continuous maps from $I$ into itself. For simplicity we shall always assume $I=[0,1]$. As will be clear from the content of this paper, this is not a significant restriction. One-dimensional and twodimensional Lebesgue measures will be denoted respectively by $\lambda$ and $\lambda^{2}$.

Let $\mathbb{N}$ be the set of positive integers. A point $p \in I$ is a periodic point of a map $f \in C(I)$ if $f^{r}(p)=p$ for some $r \in \mathbb{N}$, where $f^{0}$ is the identity map and $f^{n}=f \circ f^{n-1}$ for any $n \in \mathbb{N}$. The least integer $r$ with this property is called the period of $p$. If $x \in I$ and $\lim _{n \rightarrow \infty}\left|f^{n}(x)-f^{n}(p)\right|=0$ for some periodic point $p$ then we say that $x$ is an asymptotically periodic point of $f$. The set of asymptotically periodic points of $f$ will be denoted by $\mathrm{AP}(f)$.

Definition 1 ([LY]). Let $f \in C(I)$ and $S \subset I$. We say that $S$ is a scrambled set of $f$ if for any $x, y \in S, x \neq y$, and any periodic point $p$ of $f$ the following properties hold:

(i) $\liminf _{n \rightarrow \infty}\left|f^{n}(x)-f^{n}(y)\right|=0$;

(ii) $\lim \sup _{n \rightarrow \infty}\left|f^{n}(x)-f^{n}(y)\right|>0$;

(iii) $\lim \sup _{n \rightarrow \infty}\left|f^{n}(x)-f^{n}(p)\right|>0$.

1991 Mathematics Subject Classification: Primary 58F13; Secondary 54H20, 26A18.

Key words and phrases: chaos in the sense of Li and Yorke, dense chaos, generic chaos, full chaos, scrambled set.

This work has been partially supported by the DGICYT grant PB91-0575. 
If $f$ has an uncountable scrambled set then it is said to be chaotic in the sense of $L i$ and Yorke.

In [Sm3] it is shown that if $f \in C(I)$ then exactly one of the following alternatives must occur: either $f$ is chaotic, or all the points from $I$ are "almost" asymptotically periodic, in the sense that for any $x \in I$ and $\varepsilon>0$ there is a periodic point $p$ such $\limsup _{n \rightarrow \infty}\left|f^{n}(x)-f^{n}(p)\right|<\varepsilon$. Hence, chaoticity in the sense of $\mathrm{Li}$ and Yorke is a valuable criterion to decide whether the dynamics of $f$ is "complex" or not. However, it must be emphasized that this "complex" behaviour may be virtually "unobservable". For instance, the map $f \in C(I)$ defined by $f(x)=\alpha x(1-x), \alpha \approx 3.83187 \ldots$, has $1 / 2$ as a periodic point of period 3 and thus $f$ is chaotic by [LY]. On the other hand, $\operatorname{AP}(f)$ is a full measure residual set (see $[\mathrm{Gu}]$ ).

Hence, the question of finding a reasonable characterization of "observable" chaos (and, in particular, "complete" chaos) arises in a natural way. Of course, we could for example look for "large" scrambled sets but the problem is that, at least from a topological point of view, scrambled sets are rather small: they cannot be residual in any subinterval of $I$ (see [Ge1]). Fortunately, it is posible to construct some examples of scrambled sets of positive or even full Lebesgue measure (cf. [Ka], [Sm2], [JaS], [Sm3], [Mi], $[\mathrm{BH}],[\mathrm{Ji} 1],[\mathrm{Ji} 2])$.

Nevertheless, to identify "observable" chaos and existence of scrambled sets of positive measure may be misleading. The paradigmatic example is the "tent" map $g \in C(I)$ defined by $g(x)=1-|2 x-1|$ for any $x$. It is well known that the behaviour of $g$ is extremely complicated, even from the measure-theoretic point of view (cf. e.g. [Ji4]). On the other hand, it has no scrambled sets of positive measure (although it has a non-measurable scrambled set of full outer measure which is of the second Baire category in any subinterval of $I$ ), see [Sm1].

In [Pi1], Piórek uses for the first time an idea due to Lasota which may be very useful here. Namely, he considers the sets $\mathrm{C}_{1}(f), \mathrm{C}_{2}(f) \subset I^{2}$ given by

$$
\begin{aligned}
& \mathrm{C}_{1}(f)=\left\{(x, y) \in I^{2}: \liminf _{n \rightarrow \infty}\left|f^{n}(x)-f^{n}(y)\right|=0\right\}, \\
& \mathrm{C}_{2}(f)=\left\{(x, y) \in I^{2}: \limsup _{n \rightarrow \infty}\left|f^{n}(x)-f^{n}(y)\right|>0\right\},
\end{aligned}
$$

and defines a map $f \in C(I)$ to be generically chaotic if $\mathrm{C}(f)=\mathrm{C}_{1}(f) \cap \mathrm{C}_{2}(f)$ is residual in $I^{2}$. The notions of dense chaoticity (cf. [Sn2]) and full chaoticity can be analogously introduced, demanding now $\mathrm{C}(f)$ to be respectively a dense or a full measure set. We emphasize that $\mathrm{C}(f)$ is a Borel set (cf. [Ji4]) and hence it is always measurable.

In general, we still have a rather fragmentary information about the above notions. For the sake of completeness, we would like to recall all 
the existing bibliography we have knowledge of. Generic and dense chaos have been characterized respectively in [Sn1] and [Sn2], and it is known that dense chaos need not imply generic chaos (cf. [Sn1]). In [Sn3], the definitions of dense and generic chaos are extended to get clearer "physical" interpretations. A number of questions related with generic chaos in more general settings have been considered in [Pi1], [Pi2], [Pi3] and [Li]. The measure of the set $\mathrm{C}(f)$ has been explicitly calculated for a number of maps (see [Ge2], [BJ], [Ji3], [Ji4]). For example, it is known that $\lambda^{2}(\mathrm{C}(g))=1$ for the tent map $g$.

Dense, generic and full chaoticity can all be considered as different approaches to the idea of "complete" chaotic behaviour. Here, the question of the relations between generic and full chaos suggests itself. In general, of course, a residual set need not have full or even positive measure. However, we shall prove

TheOREM A. Let $f \in C(I)$ and suppose that $\lambda^{2}\left(\mathrm{C}_{1}(f)\right)=\lambda(I)^{2}$ and $\lambda^{2}\left(\mathrm{C}_{2}(f) \cap J^{2}\right)>0$ for any subinterval $J$ of $I$. Then $f$ it is generically chaotic. In particular, if $f \in C(I)$ is fully chaotic then it is generically chaotic.

Theorem B below will also show that a generically chaotic map may not be fully chaotic. Hence, full chaoticity could be in our opinion a reasonable definition of "complete" chaos. In this line of reasoning, we could consider chaos for $f$ to be "observable" if $\mathrm{C}(f)$ has positive measure: informally speaking, this means that for any two points chosen at random there is a positive probability that their iterates come alternatively close to and far from each other. Moreover, notice that we can easily compare now the "degree" of chaoticity of two maps $f_{1}, f_{2}$ in terms of the measures of the sets $\mathrm{C}\left(f_{1}\right)$ and $\mathrm{C}\left(f_{2}\right)$.

Observe that, when defining $\mathrm{C}(f)$, we are "forgetting" property (iii) in Definition 1 . Since asymptotically periodic points have a very regular behaviour, it seems logical to exclude them from $\mathrm{C}(f)$, because we would like $\mathrm{C}(f)$ to "contain" only the complicated part of the dynamics of $f$. More precisely, we should consider instead the set $\mathrm{Ch}(f)=\mathrm{C}(f) \backslash((\mathrm{AP}(f) \times I) \cup$ $(I \times \operatorname{AP}(f)))$, that is, the set of points $(x, y)$ (with $x \neq y)$ for which $\{x, y\}$ is scrambled. (We remark that $\mathrm{Ch}(f)$ is still a Borel set, cf. [Ji4].)

Indeed, Definition 1(iii) turns out to be rather superfluous: as is easy to check, if $S \subset I$ is a set satisfying Definition 1(i), (ii) then there is an $x \in S$ such that $S \backslash\{x\}$ is scrambled. Further, $f \in C(I)$ is fully (resp. generically, densely) chaotic if and only if $\mathrm{Ch}(f)$ is a full measure (resp. residual, dense) set. This is because if $\mathrm{Ch}(f)$ is a full measure (resp. residual, dense) set then, since $\mathrm{C}(f) \cap \operatorname{AP}(f)^{2}=\emptyset$, it follows that $\operatorname{AP}(f)$ is a measure zero set (resp. a first category set-use [Ku], Corollary 1b, p. 247-, a set with empty 
interior). This implies that $(\mathrm{AP}(f) \times I) \cup(I \times \mathrm{AP}(f))$ is also a measure zero set (resp. a first category set, a set with empty interior). Hence, $\mathrm{C}(f)$ is a full measure (resp. residual, dense) set.

It is natural to presume that, in general, $\lambda^{2}(\mathrm{C}(f))=\lambda^{2}(\mathrm{Ch}(f))$, or at least $\lambda^{2}(\mathrm{C}(f))>0$ if and only if $\lambda^{2}(\mathrm{Ch}(f))>0$. Surprisingly, this need not be the case:

TheOREM B. There is a map $f \in C(I)$ with the following properties:

(i) $f$ is generically chaotic;

(ii) $0<\lambda^{2}(\mathrm{C}(f))<\lambda(I)$;

(iii) $\lambda^{2}(\mathrm{Ch}(f))=0$.

In view of Theorem $\mathrm{B}$, we propose $\lambda(\mathrm{Ch}(f))>0$ as a plausible definition of "observable" chaos.

We shall prove Theorems A and B in the next section. Some complementary examples and concluding remarks are given in Section 3.

2. Proof of Theorems A and B. The proof of Theorem A is based on some previous lemmas. Let us first introduce some necessary notation.

Let $f \in C(I)$ and $A \subset I$. $\mathrm{Cl} A$ and $\operatorname{Int} A$ denote respectively the closure and the interior of $A$, and $\left.f\right|_{A}$ is the restriction of $f$ to $A$. The map $f$ is said to be topologically transitive if the sequence $\left(f^{n}(x)\right)_{n=0}^{\infty}$ is dense in $I$ for some $x \in I$. If $J$ is a compact subinterval of $I$, we say that $J$ is an invariant interval of $f$ if $f(J) \subset J$. If additionally $\left.f\right|_{J}$ is topologically transitive then $J$ is called transitive. We say that $\left(J_{n}\right)_{n=1}^{\infty}$ is a sequence of nested intervals of $f$ if each $J_{n}$ is a transitive interval, $J_{n+1} \subset J_{n}$ for any $n$ and $\lambda\left(J_{n}\right) \rightarrow 0$ as $n \rightarrow \infty$.

Lemma 1 ([Sh1], Theorem 1.2(h)). Let $f \in C(I)$. Then $f$ is generically chaotic if and only if the following two conditions are satisfied simultaneously:

(i) $f$ has a unique transitive interval or two transitive intervals having exactly one common point;

(ii) for any subinterval $J$ of $I$ there is a transitive interval $K$ with $\operatorname{Int} K \cap$ $\bigcup_{n=0}^{\infty} f^{n}(J) \neq \emptyset$.

Lemma 2 ([BH], Proposition 2.4). Let $f \in C(I)$. Then $f$ is topologically transitive if and only if for any subinterval $J$ of I we have $\mathrm{Cl}\left(\bigcup_{n=0}^{\infty} f^{n}(J)\right)$ $=I$.

Lemma 3. Let $f \in C(I)$ and suppose that both $\mathrm{C}_{1}(f)$ and $\mathrm{C}_{2}(f)$ are dense. Let $J$ be a subinterval of $I$. Then $\mathrm{Cl}\left(\bigcup_{n=0}^{\infty} f^{n}(J)\right)$ includes an invariant interval. 
Proof. First, there are some $n \geq 0, k>0$ with $f^{n}(J) \cap f^{n+k}(J) \neq \emptyset$. Otherwise, $\lambda\left(f^{n}(J)\right) \rightarrow 0$ as $n \rightarrow \infty$ and $\mathrm{C}_{2}(f) \cap J^{2}=\emptyset$, a contradiction.

Then $K=\operatorname{Cl}\left(\bigcup_{m=0}^{\infty} f^{n+m k}(J)\right)$ is a compact interval and $f^{k}(K) \subset K$. Let $l>0$ be minimal with the property that, for some compact interval $L \subset \mathrm{Cl}\left(\bigcup_{n=0}^{\infty} f^{n}(J)\right), f^{l}(L) \subset L$. It turns out that $L \cap f^{n}(L)=\emptyset$ for any $0<n<l$. If not, find a minimal $0<r<l$ for which $L \cap f^{r}(L) \neq \emptyset$. Clearly, $r$ divides $l$ and for the interval $L^{\prime}=\bigcup_{i=0}^{l / r} f^{i r}(L)$ we obtain $f^{r}\left(L^{\prime}\right) \subset L^{\prime}$, contradicting the minimality of $l$.

Suppose $l>1$. Then $L$ and $f(L)$ are disjoint and $\mathrm{C}_{1} \cap(L \times f(L))=\emptyset$, but simultaneously $\mathrm{C}_{2}(f) \cap L^{2} \neq \emptyset$ so $f(L)$ must be a non-degenerate interval. This is impossible. Thus $l=1$ and $L$ is the invariant interval we were looking for.

LEMMA 4. Let $f \in C(I)$ and suppose that both $\mathrm{C}_{1}(f)$ and $\mathrm{C}_{2}(f)$ are dense. Let $J \subset I$ be an invariant interval. Then $J$ includes either a transitive interval or a sequence of nested intervals.

Proof. Define $\delta=\inf \{\lambda(K): K$ is an invariant subinterval of $J\}$. Then several possibilities arise. If $\delta=\lambda(J)$ then, according to Lemmas 2 and $3, J$ itself is transitive. If $0<\delta<\lambda(J)$, construct a sequence $\left(K_{n}\right)_{n}$ of invariant subintervals of $J$ with $\lambda\left(K_{n}\right) \rightarrow \delta$ as $n \rightarrow \infty$. It is clearly not restrictive to assume that $K=\bigcap_{n} K_{n}$ contains an interval and then $K$ itself is an interval. Further, $f(K) \subset K$, so $\lambda(K)=\delta$. In particular, $K$ does not include any invariant intervals different from itself. Hence, it is transitive by Lemmas 2 and 3.

Finally, assume $\delta=0$ and fix an invariant subinterval $J_{1}$ of $J$ with $\lambda\left(J_{1}\right)<\lambda(J) / 2$. After a similar reasoning for $J_{1}$, we can find either a transitive subinterval of $J_{1}$ or an invariant subinterval $J_{2}$ of $J_{1}$ with $\lambda\left(J_{2}\right)<$ $\lambda(J) / 2^{2}$. Proceeding in this way, either we find a transitive subinterval of $J$ or we construct a sequence of nested intervals. This finishes the proof.

The following lemma is essentially similar to Lemma 4.8 of [Sn1]. For the sake of completeness, we give here the proof.

Lemma 5. Let $f \in C(I)$ and suppose that $\lambda^{2}\left(\mathrm{C}_{1}(f)\right)=\lambda(I)^{2}$ and $\lambda^{2}\left(\mathrm{C}_{2}(f) \cap J^{2}\right)>0$ for any subinterval $J$ of $I$. Then it has no sequences of nested intervals.

P r o o f. Suppose $\left(J_{n}\right)_{n=1}^{\infty}$ were a sequence of nested intervals of $f$. Clearly, we can assume that $\mathrm{Cl}\left(J_{n} \backslash J_{n+1}\right)$ and $J_{n+2}$ are disjoint for any $n$. Define $K_{n}=\left\{x \in J_{1}\right.$ : there is a $k \geq 0$ such that $\left.f^{k}(x) \in J_{n}\right\}$ for any $n$. Then $\lambda\left(K_{n}\right)=\lambda\left(J_{1}\right)$. In fact, assume that $J_{1} \backslash K_{l}$ has positive measure for some $l$. Since $f^{m}(x) \in J_{1} \backslash J_{l}$ for any $x \in J_{1} \backslash K_{l}$ and $m \geq 0$, we get $\mathrm{C}_{1}(f) \cap\left(J_{l+1} \times\left(J_{1} \backslash K_{l}\right)\right)=\emptyset$, contradicting $\lambda^{2}\left(\mathrm{C}_{1}(f)\right)=\lambda(I)^{2}$. 
Hence, the measure of $K=\bigcap_{n=1}^{\infty} K_{n}$ is $\lambda\left(J_{1}\right)$ as well. If $\{p\}=\bigcap_{n=1}^{\infty} J_{n}$ then $\lim _{m \rightarrow \infty} f^{m}(x)=p$ for any $x \in K$. This means that $\lambda^{2}\left(\mathrm{C}_{2}(f) \cap J_{1}^{2}\right)=0$; a contradiction.

Proof of Theorem A. First notice that, since $\lim \sup _{n \rightarrow \infty} \lambda\left(f^{n}(J)\right)$ $>0$ for any subinterval $J$ of $I$, we can use Remark 4.14 of [Sn1] to prove that $\mathrm{C}_{2}(f)$ is dense and so $f$ satisfies the requirements of Lemmas 3 and 4 .

Suppose that $f$ is not generically choatic. Since topologically transitive maps are generically chaotic by Lemma 1 , we can use Lemmas $2-5$ to deduce the existence of a transitive interval $K$ strictly included in $I$. Furthermore, there must exist an interval $J \subset I$ with Int $K \cap \bigcup_{n=0}^{\infty} f^{n}(J)=\emptyset$; otherwise $K$ would be the only transitive interval of $f$ (because two transitive intervals can intersect at most in one point) and (i) and (ii) of Lemma 1 would hold. According to Lemmas 3-5, we can then find a transitive interval $L$ having at most one common point with $K$. In fact, the density of $\mathrm{C}_{1}(f)$ forces $K \cap L \neq \emptyset$. Then a similar argument allows us to find a transitive interval $M$ not intersecting $\operatorname{Int}(K \cup L)$. Hence, either $M \cap K=\emptyset$ or $M \cap L=\emptyset$, both cases contradicting the density of $\mathrm{C}_{1}(f)$.

Before proving Theorem B, let us state a weaker version as an auxiliary lemma:

Lemma 6. There is a map $f \in C(I)$ with the following properties:

(i) $f$ is generically chaotic;

(ii) if a is the right endpoint of I then $\lim _{n \rightarrow \infty} f^{n}(x)=$ a for almost all $x \in I$ (in the sense of Lebesgue measure).

Proof. Let $g$ be defined by $g(x)=(x+1) / 2$ for any $x$. Fix $a_{0} \in(0,1)$ and set $a_{n}=g^{n}\left(a_{0}\right)$ and $I_{n}=\left[a_{n}, a_{n+1}\right]$ for any $n$. Also, let $A \subset I_{0}$ be a Cantor set of positive measure containing both $a_{0}$ and $a_{1}$ and let $\left\{O_{0}^{m}\right\}_{m=1}^{\infty}$ denote the family of connected components of $I_{0} \backslash A$. For any $n, m$ define $O_{n}^{m}=g^{n}\left(O_{0}^{m}\right)$ and let $c_{n}^{m}$ be the midpoint of $O_{n}^{m}$. Finally, write $B=$ $\bigcup_{n=0}^{\infty} I_{n} \backslash\left(\bigcup_{m=1}^{n+1} O_{n}^{m}\right)$ and $C=\bigcup_{n=0}^{\infty} g^{n}(A)$.

Let us now define $f$, which will consist of infinitely many consecutive linear (affine) pieces. We put $f(x)=g(x)$ for any $x \in B$. Further, we define $f(0)=0, f(1)=1, f\left(c_{n}^{m}\right)=c_{n-1}^{1}$ for any $n \geq 0$ (here we mean $c_{-1}^{1}=0$ ) and extend $f$ by linearity to the rest of $I$.

We clearly have $f \in C(I)$ and

$$
\lim _{n \rightarrow \infty} f^{n}(x)=1 \quad \text { for any } x \in C .
$$

Redefining $a_{0}$ and $A$ if necessary we can suppose that

$$
\lambda\left(f\left(J_{i}\right) \cap C\right) / \lambda\left(f\left(J_{i}\right)\right)>1 / 2 \quad \text { for any } i,
$$


where $\left\{J_{i}\right\}_{i=1}^{\infty}$ denotes the family of maximal subintervals of $I$ on which $f$ is linear. Further, we can also assume that

$$
f^{\prime}(x)>2 \quad \text { for any } x \in\left[0, a_{0}\right)
$$

and, if $n \geq 0$,

$$
\left|f^{\prime}(x)\right|>2^{n+1} \quad \text { for any } x \in O_{n}^{m} \backslash\left\{c_{n}^{m}\right\}, m=1, \ldots, n+1 .
$$

We prove that $f$ is appropriate for our purposes. First, observe that each $f\left(J_{i}\right) \backslash C$ can be written as a countable union of intervals with pairwise disjoint interiors, in such a way that if $J$ is one of these intervals then there is a $k \geq 0$ such that $\left.f^{k}\right|_{J}$ is linear and $f^{k}(J)=J_{l}$ for some $l$. Hence, (1) and (2) imply that $\lim _{n \rightarrow \infty} f^{n}(x)=1$ for almost all $x \in I$.

On the other hand, let $J$ be an arbitrary subinterval of $I$. By (3) and (4) (recall also that $g^{\prime}(x)=1 / 2$ for any $x$ ), there is a $k \geq 0$ such that $f^{k}(J) \not \subset J_{i}$ for any $i$, and then an $l \geq k$ such that $f^{l}(J) \supset J_{j}$ for some $j$. From this, $\mathrm{Cl}\left(\bigcup_{n=0}^{\infty} f^{n}(J)\right)=I$. Then $f$ is generically chaotic by Lemmas 1 and 2 .

Proof of Theorem B. We shall construct a generically chaotic map $f \in C(I)$ for which $\lambda^{2}(\mathrm{C}(f))=1 / 2$ and $\lambda^{2}(\mathrm{Ch}(f))=0$. The map $f$ will be topologically semiconjugate to the tent map $g(x)=1-|2 x-1|$, that is, for an appropriate monotone onto map $h \in C(I)$ we shall have $h \circ f=g \circ h$.

The first step of our construction will be to define a set $C \subset I$ with some special properties with respect to $g$. For this purpose we need some auxiliary notation. Define

$$
N_{n}=\left\{(2 m-1) 2^{n-1}: m \in \mathbb{N}\right\}
$$

for any $n \in \mathbb{N}$. Note that $N_{n} \cap N_{k}=\emptyset$ if $n \neq k$ and $\bigcup_{n=1}^{\infty} N_{n}=\mathbb{N}$. Namely $N_{1}=\{1,3,5,7,9, \ldots\}, N_{2}=\{2,6,10,14,18, \ldots\}, N_{3}=\{4,12,20,28,36, \ldots\}$ and so on. Next, let also

$$
M_{1}^{n}=\left\{\frac{n(n-1)}{2}+i: 1 \leq i \leq n\right\}
$$

for any $n \in \mathbb{N}$. As before we have $M_{1}^{n} \cap M_{1}^{k}=\emptyset$ if $n \neq k$ and $\bigcup_{n=1}^{\infty} M_{1}^{n}=\mathbb{N}$. For example $M_{1}^{1}=\{1\}, M_{1}^{2}=\{2,3\}, M_{1}^{3}=\{4,5,6\}, M_{1}^{4}=\{7,8,9,10\}$ and so on. Finally, define inductively

$$
M_{m+1}^{n}=M_{m}^{2 n-1} \cup M_{m}^{2 n}
$$

for any $m, n \in \mathbb{N}$.

Recall that we can associate with any $x \in I$ its extended itinerary $\iota_{g}(x) \in$ $\{0,1,2\}^{\infty}$ (or simply $\iota(x)$ ) defined for any $n \geq 1$ by

$$
\iota(x)_{n}= \begin{cases}0 & \text { if } g^{n-1}(x)<1 / 2, \\ 1 & \text { if } g^{n-1}(x)=1 / 2, \\ 2 & \text { if } g^{n-1}(x)>1 / 2,\end{cases}
$$


(of course $\iota(x)_{n}$ denotes the $n$th term of $\iota(x)$ ). As is well known (use e.g. [CE], Theorem II.3.8), for any $\alpha \in\{0,2\}^{\infty}$ with the property that there is no $k$ such that $\alpha_{n}=0$ for any $n>k$, there is exactly one $x \in I$ such that $\iota(x)=\alpha$. We shall use these extended itineraries to define $C$. Namely, for any $j \in\{0,2\}$ let $C_{j}$ be the set of points $x \in I$ such that, for any $n$,

$$
\iota(x)_{n}= \begin{cases}j & \text { if } n \in N_{1} \cap M_{1}^{k} \text { for some even number } k, \\ 2-j & \text { if } n \in N_{1} \cap M_{1}^{k} \text { for some odd number } k .\end{cases}
$$

Inductively, suppose that the sets $C_{\alpha} \subset I$ have already been defined for any $\alpha \in\{0,2\}^{i}$. Then, if $\beta \in\{0,2\}^{i}$ and $j \in\{0,2\}$, let $C_{\beta, j}$ denote the set of points $x \in C_{\beta}$ with the property that, for any $n$,

$$
\iota(x)_{n}= \begin{cases}j & \text { if } n \in N_{i+1} \cap M_{i+1}^{k} \text { for some even number } k, \\ 2-j & \text { if } n \in N_{i+1} \cap M_{i+1}^{k} \text { for some odd number } k .\end{cases}
$$

For instance, if $x \in C_{0,0}$ then we must have

$$
\iota(x)=2 ; 2,0 ; *, 2,0 ; 0, *, 0,0 ; 2, *, 2,2,2 ; *, 0,2,0, *, 0 ; 0,2, *, 2,0,2, * ; \ldots,
$$

while if $x \in C_{2,0}$ then

$$
\iota(x)=0 ; 2,2 ; *, 0,0 ; 2, *, 2,0 ; 0, *, 0,2,0 ; *, 2,2,2, *, 2 ; 0,0, *, 0,0,0, * ; \ldots
$$

(here the semicolons mark the $M_{1}^{n}$ blocks, while the symbol "*" denotes indistinctly 0 or 2 ).

In this way we have defined the sets $C_{\alpha}$ for any $m \in \mathbb{N}$ and $\alpha \in\{0,2\}^{m}$. Finally, we put

$$
C=\bigcap_{m=1}^{\infty} \bigcup_{\alpha \in\{0,2\}^{m}} C_{\alpha}
$$

Let us describe some useful properties of $C$. To begin with,

$C$ is a Cantor set.

In fact, each $C_{\alpha}$ is obviously non-empty, and it is not difficult to check that it is closed as well. Further, $C$ does not intersect the set $D$ of the preimages of 0 , which is clearly dense in $I$, so Int $C=\emptyset$. Finally, $C$ has no isolated points, because if $x, y \in I$ and $\iota(x)_{n}=\iota(y)_{n}$ for any $1 \leq n \leq m$ then it is easy to check that $|y-x|<1 / 2^{m}$.

On the other hand, let $x, y \in C, x \neq y$. Then there is a $k \in \mathbb{N}$ such that (6) either $g^{n-1}(x)<1 / 2<g^{n-1}(y)$ or $g^{n-1}(y)<1 / 2<g^{n-1}(x)$

for any $n \in N_{k}$ : just choose $k$ with the property that $x \in C_{\alpha}, y \in C_{\beta}$ for some $\alpha, \beta \in\{0,2\}^{k}$ with $\alpha_{k} \neq \beta_{k}$.

Finally, we claim

$$
\begin{gathered}
\limsup _{n \rightarrow \infty} g^{n}(x) \geq 1 / 2, \\
\liminf _{n \rightarrow \infty} g^{n}(x)=0,
\end{gathered}
$$


for any $x \in C$. (7) is immediate, because if $x \in C$ then the sequence $\iota(x)$ has an infinite number of $2 \mathrm{~s}$. To prove (8) it is clearly sufficient to show that for any given $x \in C$ and $k \in \mathbb{N}$ there is an $l$ as large as desired so that $\iota(x)_{l+i}=0$ for any $1 \leq i \leq k$. Take $m$ large enough with $k<2^{m}$ and find $\alpha \in\{0,2\}^{m}$ with $x \in C_{\alpha}$. Choose now some positive integers $i(j), 1 \leq j \leq m$, with $M_{j}^{i(j)} \subset M_{j+1}^{i(j+1)}$ for any $1 \leq j<m$ and such that $i(j)$ is even (resp. odd) if $\alpha_{j}=0$ (resp. $\alpha_{j}=2$ ). (The easiest way to define these numbers is to choose firstly $i(m)$, then $i(m-1)$ and so on.) Observe that if $r \in M_{1}^{i(1)}$ and is not a multiple of $2^{m}$ then $\iota(x)_{r}=0$ (because $N \backslash \bigcup_{j=1}^{m} N_{j}=\left\{n 2^{m}: n \in \mathbb{N}\right\}$ ). Further, the number $i(1)$ can be as large as necessary. This implies (8).

Next, we define for any $x \in I$ a (possibly degenerate) closed interval $I_{x} \subset I$ as follows. Construct a homeomorphism $\varphi \in C(I)$ with the property that

$$
A=\varphi(C) \text { has measure } 1 / 2
$$

(this is possible by (5)). Then put $I_{x}=\{\varphi(x)\}$ if $x \in C$. For the rest of the points $x$, the definition of $I_{x}$ is more complicated. Let $\left\{O_{n}\right\}_{n=1}^{\infty}$ be the family of connected components of $I \backslash A$. Rewrite $D$ as $\bigcup_{n=1}^{\infty}\left\{d_{n, i}\right\}_{i=1}^{\infty}$, where $\left\{d_{n, i}\right\}_{i=1}^{\infty}=D \cap \varphi^{-1}\left(O_{n}\right)$ for any $n$. Also, define for any $n$ a sequence $\left(s_{n, i}\right)_{i=1}^{\infty}$ of positive real numbers with

$$
\sum_{i=1}^{\infty} s_{n, i}=\lambda\left(O_{n}\right)
$$

We can now define $I_{x}$ for any $x \in I \backslash C$. Let $n$ be such that $\varphi(x) \in O_{n}$ and let $c_{n}$ denote the left endpoint of $O_{n}$. Then define

$$
a_{x}=c_{n}+\sum_{i: d_{n, i}<x} s_{n, i}, \quad b_{x}=c_{n}+\sum_{i: d_{n, i} \leq x} s_{n, i},
$$

and let $I_{x}$ be the convex hull of $\left\{a_{x}, b_{x}\right\}$. Observe that all the numbers $a_{x}$, $b_{x}$ are well defined except $a_{0}$, whose definition makes no sense; in this case put $a_{0}=0$.

We enumerate the relevant properties of the intervals $I_{x}$. First, clearly

$$
I_{x} \text { is non-degenerate if and only if } x \in D \text {. }
$$

Further,

$$
\sum_{x \in D} \lambda\left(I_{x}\right)=1 / 2
$$

by (10). Since $D$ is dense in $I$,

$$
x<y \text { if and only if } u<v \text { for any } u \in I_{x}, v \in I_{y}
$$


(use also (10)). Finally, we claim that

$$
\bigcup_{x \in I} I_{x}=I
$$

In fact, recall that $A \subset \bigcup_{x \in I} I_{x}$. Now, let $y \in O_{n}$ for some $n$. Of course we can assume that $y \notin I_{x}$ for any $x \in D$. Consider the set $N=\{i: u<y$ for any $\left.u \in I_{d_{n, i}}\right\}$. By $(10), c_{n}+\sum_{i \in N} s_{n, i}=y$. Take $x=\sup _{i \in N} d_{n, i}$. Clearly, $x>d_{n, i}$ for any $i \in N$. Hence, $I_{x}=\{y\}$.

We are ready to define $f$. Namely, we construct $f$ in such a way that $f\left(I_{x}\right)=I_{g(x)}$ for any $x \in I$. Further, $f$ is linear on each of the intervals $I_{x}, x \in D$, except for $I_{1 / 2}$, where it consists of two linear pieces, and $I_{0}$, where $f$ is defined in such a way that $\left.f\right|_{I_{0}} \in C\left(I_{0}\right)$ is generically chaotic and $\lim _{n \rightarrow \infty} f^{n}(x)=b_{0}$ for almost all $x \in I_{0}$. By (11), (13), (14) and Lemma 6, such a map clearly exists.

Let us check that $f$ has the desired properties. Write $B=\bigcup_{x \in D} I_{x}$. Since $B$ is dense in $I$, the definition of $f$ on the intervals $I_{x}, x \in D$, clearly implies that $f$ is generically chaotic (use Lemma 1 ) and $\lim _{n \rightarrow \infty} f^{n}(x)=b_{0}$ for almost all $x \in B$. On the other hand, (6) implies that $\liminf _{n \rightarrow \infty} \mid f^{n}(x)-$ $f^{n}(y) \mid>0$ for any $x, y \in A, x \neq y$. Since $A \cap B=\emptyset$ and $\lambda(A)+\lambda(B)=1$ (see (9) and (12)), we get

$$
\lambda^{2}(\mathrm{Ch}(f))=0 .
$$

Finally, we have $\liminf _{n \rightarrow \infty}\left|f^{n}(x)-b_{0}\right|=0$ and $\limsup _{n \rightarrow \infty}\left|f^{n}(x)-b_{0}\right| \geq$ $b_{1 / 2}-b_{0}$ for any $x \in A$ by (7) and (8). Hence,

$$
\lambda^{2}(\mathrm{C}(f))=2 \lambda(A) \lambda(B)=1 / 2 .
$$

This finishes the proof.

3. Final remarks. 1) In connexion with Theorem A, we emphasize that it is possible to construct a non-fully chaotic map $f \in C(I)$ for which $\lambda^{2}\left(C_{1}(f)\right)=\lambda(I)^{2}$ and $\lambda^{2}\left(\mathrm{C}_{2}(f) \cap J^{2}\right)>0$ for any subinterval $J$ of $I$. The map $f$ can be defined in such a way that it is topologically conjugate to the tent map $g$, that is, $h \circ f=g \circ h$ for an appropriate homeomorphism $h$.

Let us give a sketch of the construction of $f$. First, we define the sets $A, B$ by

$$
\begin{aligned}
A=\{x \in I: & \iota(x)_{n}=0 \text { for any } n \neq 2^{m}, 2^{m}-1, m \in \mathbb{N}, \\
& \iota(x)_{2^{m}}=2 \text { for any } m \in \mathbb{N}, \\
& \text { and there is an } \alpha \in\{0,2\}^{\infty} \text { such that } \\
& \left.\iota(x)_{2^{m}-1}=\alpha_{m} \text { for any } m \in \mathbb{N}\right\},
\end{aligned}
$$


and

$$
\begin{aligned}
B=\{x \in I: & \iota(x)_{n}=0 \text { for any } n \neq 2^{m}-1, m \in \mathbb{N}, \\
& \text { and there is a } y \in C \text { such that } \\
& \left.\iota(x)_{2^{m}-1}=\iota(y)_{m} \text { for any } m \in \mathbb{N}\right\}
\end{aligned}
$$

(the sequences $\iota(x)$ and the set $C$ were defined in the proof of Theorem B).

Both $A$ and $B$ are Cantor sets, and we have

$$
\begin{gathered}
A^{2} \subset \mathrm{C}_{1}(g) \backslash \mathrm{C}_{2}(g), \\
(A \times B) \cup(B \times A) \cup\left(B^{2} \backslash\{(x, x): x \in B\}\right) \subset \mathrm{C}(g) .
\end{gathered}
$$

Next, we find for any $i \in \mathbb{N}$ a family $\left\{B_{i}^{m}\right\}_{m=1}^{m(i)}$ of pairwise disjoint Cantor sets with the following properties for any given $i$ and $r \in\{1, \ldots, m(i)\}$ :

(i) There is a connected component $O$ of $I \backslash\left(\bigcup_{j=0}^{i-1} \bigcup_{m=1}^{m(j)} B_{j}^{m}\right)$ such that $A_{i}^{r} \subset O$ (here $B=B_{0}^{1}$ and $m(0)=1$ ).

(ii) There is an $l \in \mathbb{N}$ such that $f^{l}\left(B_{i}^{r}\right)=f^{l}(B)$.

(iii) For any $x \in I$ there is a $y \in \bigcup_{m=1}^{m(i)} B_{i}^{m}$ such that $|x-y|<1 / i$.

Put $B^{\prime}=\bigcup_{j=0}^{\infty} \bigcup_{m=1}^{m(j)} B_{j}^{m}$ and observe that $A \cap B^{\prime}=\emptyset$. Then it is possible to construct a homeomorphism $h: I \rightarrow I$ with the properties $\lambda\left(h^{-1}(A)\right)>0, \lambda\left(h^{-1}\left(B_{i}^{m}\right)\right)>0$ for any $i \geq 0$ and $m=1, \ldots, m(i)$, and $\lambda\left(h^{-1}(A)\right)+\lambda\left(h^{-1}\left(B^{\prime}\right)\right)=\lambda(I)$. It is sufficient to define $f=h^{-1} \circ g \circ h$.

2) We conjecture that it is possible to construct some differentiable or even $C^{\infty}$ maps with properties (i)-(iii) of Theorem B. On the other hand, let $P(I)$ denote the set of piecewise monotone maps from $C(I)$ (with a finite number of pieces). It turns out that (i)-(iii) can simultaneously hold for no map $f \in P(I)$. The reason is that if $f \in P(I)$ is densely chaotic then it is not difficult to show that $\operatorname{AP}(f)$ is countable. Hence, $\lambda^{2}(\mathrm{C}(f))=\lambda^{2}(\mathrm{Ch}(f))$.

It is known that a piecewise monotone map is densely chaotic if and only if it is generically chaotic (see [Sn2]). However, we can change slightly the construction of the map $f$ from 1 ) to get a generically chaotic map $f_{1} \in P(I)$ (topologically conjugate to the tent map) for which $\lambda^{2}\left(\mathrm{C}\left(f_{1}\right)\right)=0$. Also, there exists a map $f_{2} \in P(I)$ with $\lambda^{2}\left(\mathrm{C}\left(f_{2}\right)\right)>\lambda^{2}\left(\mathrm{Ch}\left(f_{2}\right)\right)=0$. To construct it, just consider the map $f$ and the point $b_{0}$ defined in the proof of Theorem B and put

$$
f_{2}(x)= \begin{cases}\left(x / b_{0}\right)^{1 / 2} & \text { if } x \leq b_{0}, \\ f(x) & \text { if } x>b_{0} .\end{cases}
$$

It would be very interesting to know whether it is possible to find some maps with similar properties to those of $f_{1}$ and $f_{2}$ in more "natural" settings, namely, those of piecewise linear (with finitely many pieces) or analytic maps. According to some recent results ([Ji3], [JiS], [Ji4], [MT], [AJS]), it seems reasonable to conjecture that the notions of dense and full chaoticity 
are equivalent for piecewise linear maps, and in this case both $\mathrm{C}(f)$ and $\mathrm{Ch}(f)$ have the same measure. For analytic, or even polynomial maps, things may not be that simple. With regard to this see [BKNS], where a polynomial topologically transitive map $f$ is constructed with the property that the set of limit points of the sequence $\left(f^{n}(x)\right)_{n=0}^{\infty}$ is a Cantor set for almost all $x$.

Acknowledgements. I would like to thank J. Smítal and L'. Snoha for a useful discussion about a previous version of Theorem B.

\section{References}

[AJS] L. Alsedà, V. Jiménez López and L'. Snoha, On 1-difactors of Markov graphs and the prevalence of simple solenoids, preprint, 1995.

[BJ] F. Balibrea and V. Jiménez López, A structure theorem for $C^{2}$ functions verifying the Misiurewicz condition, in: Proceedings of the European Conference on Iteration Theory (ECIT 91), Lisbon, 1991, World Sci., Singapore, 1992, 12 21.

[BH] A. M. Bruckner and T. Hu, On scrambled sets and chaotic functions, Trans. Amer. Math. Soc. 301 (1987), 289-297.

[BKNS] H. Bruin, G. Keller, T. Nowicki and S. van Strien, Absorbing Cantor sets in dynamical systems: Fibonacci maps, preprint Stony Brook 1994/2.

[CE] P. Collet and J. P. Eckmann, Iterated Maps on the Interval as Dynamical Systems, Progr. Phys. 1, Birkhäuser, Boston, 1980.

[Ge1] T. Gedeon, There are no chaotic mappings with residual scrambled sets, Bull. Austral. Math. Soc. 36 (1987), 411-416.

[Ge2] - Generic chaos can be large, Acta Math. Univ. Comenian. 54/55 (1988), $237-241$.

[Gu] J. Guckenheimer, Sensitive dependence on initial conditions for one-dimensional maps, Comm. Math. Phys. 70 (1979), 133-160.

[JaS] K. Janková and J. Smítal, A characterization of chaos, Bull. Austral. Math. Soc. 34 (1986), 283-292.

[Ji1] V. Jiménez López, $C^{1}$ weakly chaotic functions with zero topological entropy and non-flat critical points, Acta Math. Univ. Comenian. 60 (1991), 195-209.

[Ji2] - Large chaos in smooth functions of zero topological entropy, Bull. Austral. Math. Soc. 46 (1992), 271-285.

[Ji3] - , Paradoxical functions on the interval, Proc. Amer. Math. Soc. 120 (1994), 465-473.

[Ji4] - Order and chaos for a class of piecewise linear maps, Internat. J. Bifur. Chaos Appl. Sci. Engrg. 5 (1995), 1379-1394.

[JiS] V. Jiménez López and L'. Snoha, There are no piecewise linear maps of type $2^{\infty}$, preprint, 1994 .

[Ka] I. Kan, A chaotic function possessing a scrambled set of positive Lebesgue measure, Proc. Amer. Math. Soc. 92 (1984), 45-49.

[Ku] K. Kuratowski, Topology, Vol. I, Academic Press, New York, 1966.

[LY] T.-Y. Li and J. A. Yorke, Period three implies chaos, Amer. Math. Monthly 82 (1975), 985-992.

[Li] G.-F. Liao, A note on generic chaos, Ann. Polon. Math. 59 (1994), 99-105. 
[MT] M. Martens and C. Tresser, Forcing of periodic orbits and renormalization of piecewise affine maps, preprint Stony Brook 1994/17.

[Mi] M. Misiurewicz, Chaos almost everywhere, in: Iteration Theory and its Functional Equations, Lecture Notes in Math. 1163, Springer, Berlin, 1985, 125-130.

[Pi1] J. Piórek, On the generic chaos in dynamical systems, Univ. Iagell. Acta Math. 25 (1985), 293-298.

[Pi2] -, On generic chaos of shifts in function spaces, Ann. Polon. Math. 52 (1990), 139-146.

[Pi3] -, On weakly mixing and generic chaos, Univ. Iagell. Acta Math. 28 (1991), $245-250$.

[Sm1] J. Smítal, A chaotic function with some extremal properties, Proc. Amer. Math. Soc. 87 (1983), 54-56.

[Sm2] -, A chaotic function with a scrambled set of positive Lebesgue measure, ibid. 92 (1984), 50-54.

[Sm3] -, Chaotic functions with zero topological entropy, Trans. Amer. Math. Soc. 297 (1986), 269-282.

[Sn1] L'. Snoha, Generic chaos, Comment. Math. Univ. Carolin. 31 (1990), 793-810.

[Sn2] -, Dense chaos, ibid. 33 (1992), 747-752.

[Sn3] —, Two-parameter chaos, Acta Univ. M. Belii 1 (1993), 3-6.

Departamento de Matemáticas

Universidad de Murcia

Campus de Espinardo

Aptdo. de Correos 4021

30100 Murcia, Spain

E-mail: vjimenez@fcu.um.es 